\title{
Editorial
}

\section{Breast cancer: Are we in the best era?}

Breast cancer has intrigued humanity from time immemorial. Arriving silently in the life of a woman with the face of innocent lump to rapidly transforming into a fatal demon, breast cancer could not be stopped until the $18^{\text {th }}$ century. In fact, the first records of breast cancer can be traced in ancient Egyptian literature dating back to $1500 \mathrm{BC} \cdot{ }^{[1]}$

In 1894, William Halsted came up the theory that breast cancer spreads through surrounding tissues and lymph nodes, this led to the development of radical mastectomy as a treatment option for the disease and helped with prolonging life of the patients. However, the associated side effects and cosmetic disfigurement majorly affected the quality of life.

In the middle of $20^{\text {th }}$ century, researchers established that ovarian function and hormonal status had a great impact on the treatment. Keeping this in mind, various treatment options such as high dose irradiation of the ovaries, oophorectomy, and adrenalectomy were undertaken as a measure to improve life expectancy.

In the 1970s, modified radical mastectomy in early breast cancer was observed to be associated with reduced pain and speedy recovery. Later, lumpectomy followed by radiation was found to be equally effective in women with the early disease with breast conservation. ${ }^{[2]}$ Rapid evolution of radiotherapy from conventional to intensity-modulated radiation therapy and image-guided radiation therapy made more targeted radiotherapy delivery possible. ${ }^{[3]}$

Introduction of tamoxifen in 1977 was a major breakthrough and changed the treatment outcome. It showed a survival benefit when given with chemotherapy in early breast cancer patients. Subsequently, it was seen to be associated with a significant reduction in the recurrence and mortality in women with hormone positive cancers. The adverse effect profile of the drug constituted increased incidence of endometrial carcinoma and deep vein thrombosis, but the benefits outweighed the harms. ${ }^{[4]}$ Aromatase inhibitors such as letrozole were found to be even more effective in risk reduction than tamoxifen in postmenopausal women.

In the 1980s, regular use of mammography for screening resulted in early detection and substantial decrease in mortality due to breast cancer. Later, it was found that digital mammography is a better test. ${ }^{[5]}$
Chemotherapeutic agent doxorubicin demonstrated tumor shrinkage in advanced disease and is still considered the mainstay of treatment in combination chemotherapy. Taxanes emerged as an effective option in the treatment of advanced disease. They showed better survival when added to other chemotherapeutic agents. The first monoclonal antibody targeting human epidermal growth factor 2 (HER2)/neu receptor, trastuzumab was introduced in 1998, dramatically increased survival in HER2 positive advanced disease.

Discovery of BRCA1 and BRCA2 gene mutation as risk factors led to frequent screening in this subset of populations and to undergo preventive surgical breast and/or ovary removal. Use of drugs such as tamoxifen or raloxifene for preventive purposes was also found to be effective.

Oncotype DX, a gene test introduced in the early 2000s, helped clinicians to identify the subset of patients requiring additional chemotherapy to prevent recurrence and hence sparing remainder of the patients from unnecessary side effects and economic burden.

With the advancements of tumor biology understanding, specific pathway inhibitors with promising results came up. ${ }^{[6]}$ Since the beginning of this century, a variety of drugs with a spectrum of activities have come up.

Over the last decade, we have found many new agents. Lapatinib was approved for the treatment in patients not responding to trastuzumab. Epithelone class drug ixabepilone emerged as a treatment in patients whose advanced disease was unresponsive to any chemotherapy. Antivascular endothelial growth factor monoclonal antibody bevacizumab was granted approval for the use with paclitaxel in newly diagnosed patients. Eribulin mesylate studies reflected prolongation of survival in chemorefractory cases. PARP inhibitors demonstrated promising results in difficult to treat cases. Denosumab was shown to delay bone metastasis.

The HER2 positive tumors are being better managed with the addition of Pertuzumab and T-DM1. The hormone positive patients have new drugs such as mTOR inhibitor everolimus and CDK inhibitor palbociclib producing durable responses. ${ }^{[7]}$ Many more exciting molecules are in Phase II and III trials. 
In the current scenario, advances in screening, as well as treatment with chemotherapy, hormonal therapy, targeted therapy, surgery, and node evaluation, has led to a higher cure rates and substantial survival benefit among the rest. Added to this, the molecular and genetic profiling has pointed out the differences in prognosis and response to treatment in different subtypes of cancers.

Breast cancer has emerged as the evidence of victory of human will and endurance in the fight against evil, even when the enemy lies within. The progress in the breast cancer inspires us to replicate the success story in other tumors.

We have many more frontiers to be conquered; still we are surely entering the best era!

\section{C. Doval}

Department of Medical Oncology, Rajiv Gandhi Cancer Institute, New Delhi, India

Address for correspondence: Dr. D. C. Doval, Department of Medical Oncology, Rajiv Gandhi Cancer Institute, New Delhi - 110 085, India. E-mail: doval.dc@rgcirc.org

\section{References}

1. Breasted JH. The Edwin Smith Surgical Papyrus. Translation for the New York Historical Society. Chicago, IL: University of Chicago Press; 1930.

2. Veronesi U, Cascinelli N, Mariani L, Greco M, Saccozzi R,
Luini A, et al. Twenty-year follow-up of a randomized study comparing breast-conserving surgery with radical mastectomy for early breast cancer. N Engl J Med 2002;347:1227-32.

3. Bartelink H, Horiot JC, Poortmans P, Struikmans H, Van den Bogaert W, Barillot I, et al. Recurrence rates after treatment of breast cancer with standard radiotherapy with or without additional radiation. N Engl J Med 2001;345:1378-87.

4. Baum M, Brinkley DM, Dossett JA, McPherson K, Patterson JS, Rubens RD, et al. Improved survival among patients treated with adjuvant tamoxifen after mastectomy for early breast cancer. Lancet $1983 ; 2: 450$.

5. Verbeek AL. Mammographic screening: Keeping women alive. Womens Health (Lond Engl) 2011;7:631-3.

6. Ellis MJ, Perou CM. The genomic landscape of breast cancer as a therapeutic roadmap. Cancer Discov 2013;3:27-34.

7. Asghar U, Witkiewicz AK, Turner NC, Knudsen ES. The history and future of targeting cyclin-dependent kinases in cancer therapy. Nat Rev Drug Discov 2015;14:130-46.

This is an open access article distributed under the terms of the Creative Commons Attribution-NonCommercial-ShareAlike 3.0 License, which allows others to remix, tweak, and build upon the work non-commercially, as long as the author is credited and the new creations are licensed under the identical terms.

\begin{tabular}{|l|c|}
\hline \multicolumn{2}{|c|}{ Access this article online } \\
Website: & Quick Response Code \\
www.asjo.in & \\
\hline &
\end{tabular}

How to cite this article: Doval DC. Breast cancer: Are we in the best era?. Asian J Oncol 2015;1:63-4. 